\title{
Time evolution of medium and long-period ground tilting at Campi Flegrei caldera
}

\author{
Simona Petrosino ${ }^{1}$, Ciro Ricco ${ }^{1}$, Enza De Lauro ${ }^{2}$, Ida Aquino ${ }^{1}$, and Mariarosaria Falanga ${ }^{2}$ \\ ${ }^{1}$ Istituto Nazionale di Geofisica e Vulcanologia, Sezione di Napoli - Osservatorio Vesuviano, Naples, Italy \\ ${ }^{2}$ Università degli Studi di Salerno, Dipartimento di Ingegneria dell'Informazione ed Elettrica e \\ Matematica applicata/DIEM, Fisciano, Italy
}

Correspondence: Ida Aquino (ida.aquino@ingv.it)

Received: 24 September 2019 - Revised: 14 January 2020 - Accepted: 30 January 2020 - Published: 5 March 2020

\begin{abstract}
We analyse tiltmeter time series recorded from April 2015 to March 2019 at three borehole instruments installed at Campi Flegrei caldera (Italy). We evaluate the crustal response in terms of ground tilting to external excitations of medium/long-period tidal constituents by applying a polarization analysis. The azimuths of the tilt vectors show well-defined polarization directions and the ground tilting planes oscillate with the periodicity of the corresponding tidal constituents. For two of the three tiltmeters, the average ground oscillation pattern related to the monthly $\mathrm{Mm}$ and fortnightly Mf constituents show seasonal variations, which can be ascribed to rainfall-induced tilting. In addition, for the same two instruments, a clear seasonal amplitude modulation of the diurnal S1 constituent appears in the time series, revealing the occurrence of site thermoelastic effects. The results indicate that the tidal tilting is mainly controlled by the local stress field distribution and rheology; in addition, seasonal exogenous factors like rainfalls play a role in modulating the ground deformation.
\end{abstract}

\section{Introduction}

Geophysical signals are a superposition of many effects related to different sources, which act over several time scales, from seconds/hours up to seasonal/annual. In particular, ground deformation time series contain distinct components ascribable to both endogenous, like tectonic stresses, magmatic mass movement, hydrothermal fluid migration, and exogenous mechanisms, like solid earth tides, rainfall and hydrological factors, atmospheric pressure variations, thermoelastic strain (Weise et al., 1998; Dong et al., 2002; Wester- haus et al., 2008; Ben-Zion and Allam, 2013). In many cases, external phenomena can induce a departure of the deformation trend generated by internal sources and eventually mask interesting signals related solely to the intrinsic dynamics (Dal Moro and Zadro, 1998). Thus, it is important to identify the external contributions in the time series and remove them. Otherwise, the analysis of the deformation induced by exogenous components can be exploited to obtain useful indications on the shallow structure of the medium and its rheology, as well as on the crustal stress state (Queitsch et al., 2014; Lesparre et al., 2017; De Lauro et al., 2018). Sometimes the mixing between exogenous and endogenous factors is so complex that the external causes can even influence the internal processes (McNutt and Beavan, 1981; De Martino et al. 2011a, 2011b; De Lauro et al., 2012, 2013; Petrosino et al., 2018; Ricco et al., 2019; Cusano et al., 2020). As example, recent studies have highlighted that earth tides modulate the occurrence of long-period volcanic earthquakes (De Lauro et al., 2012) and volcano-tectonic seismicity (Petrosino et al., 2018) at Campi Flegrei, thus evidencing that exogenous phenomena play a role in the dynamics of the area.

Often, ground deformation induced by external sources show a nearly cyclic behaviour on both short (semidiurnal, diurnal) and medium/long (fortnightly, monthly, seasonal and annual) terms. Thus, the period of recurrence of a particular pattern in the ground deformation time series gives us clues about the possible generation mechanism. As example, earth tides induce ground tilt oscillations over diurnal, fortnightly and monthly time scales (Dong et al., 2002; De Lauro et al., 2018). Instead, seasonal trend in ground deformation pattern commonly observed in strain and tilt signals are mainly caused by water infiltration after the rainfalls 
(Wyatt and Berger, 1980; Kumpel et al., 2001; Uhlemann et al., 2016; Lesparre et al., 2017). Rainfall-induced tilt has been explained in terms of a variety of mechanisms, e.g. surface loading essentially due to compression of the ground by the added water mass (Kumpel et al., 2001; Westerhaus and Welle, 2002; Westerhaus et al., 2008; Lesparre et al., 2017), thermoelastic strain related by changes in ground temperature caused by rain (Kumpel et al., 2001), consolidation phenomena of sedimentary layers (Fabian and Kumpel, 2003). However, the most relevant contribution to rainfall-induced tilt is related to the process of water infiltration, which modifies the pore pressure of the medium. Ground deformation caused by changes of the pore pressure is generally predominant over other generation mechanisms, whose effects can be negligible or become relevant only in case of particular geometry such as sloping surfaces (Westerhaus and Welle, 2002; Westerhaus et al., 2008; Lesparre et al., 2017). The physical process at the origin of ground deformation related to water infiltration is explained in terms of the theory of poroelasticity (Wang, 2000; Wang and Kumpel 2003). According to this model, the tilt amplitude is proportional to the pore pressure gradient and depends on the rock poroelastic parameters. In this framework, the role of fractures and structural inhomogeneities is crucial because the water supplied by rainfalls can accumulate in discontinuities, with the effect of changing the local stress and producing fluid-induced tilt (Lesparre et al., 2017). In this case, the magnitude of the tilt and the orientation of the tilting plane can put constraints on the source location and help in identifying the hydrologically active structures. Interesting results are those of Lesparre et al. (2017), who modelled the observed rainfall-induced tilt considering different possible processes associated with water infiltration and pressure changes on fracture walls. In particular, by adopting different starting models (loading of a dry fracture or of a system of fractures, water increase in a partially flooded fracture), the authors were able to retrieve some physical parameters associated with the source as well as the estimate of the water amount involved in the process. Similarly, Weise et al. (1998) showed that even small fissures can modify pore pressure induced stresses: tiltmeter data are particularly sensitive to these variations and their analysis is useful in determining rock properties and the response to stress changes.

In the present paper, we analyse four years of tiltmeter time series recorded at Campi Flegrei from 1 April 2015 to 31 March 2019, with particular attention to the effects induced by external sources on the deformation trend. We track the time evolution of the polarization direction of the tilt vector associated to the diurnal (S1), lunisolar fortnightly (Mf) and lunar monthly $(\mathrm{Mm})$ tidal constituents. We find evidence that the deformation pattern over medium/long period time scales shows precise polarization directions, which at some sites are affected by seasonal variations related to rainfall. In addition, depending on the site, seasonality also modulates the amplitude of the tilt induced by the diurnal S1 constituent indicating the occurrence of thermoelastic effects.

\section{The Tiltmeter Network at Campi Flegrei}

Campi Flegrei caldera is located to the West of Naples (Southern Italy) and it is considered one of the most dangerous volcanic complex in the world. Its most relevant feature is the phenomenon of bradyseism (Del Gaudio et al., 2010) which consists of a slow subsidence alternated to fast ground uplifts (De Martino et al., 2014) accompanied by seismicity (Saccorottti et al., 2007; Falanga and Petrosino 2012; De Lauro et al. 2016; Capuano et al., 2017). Due to the high volcanic risk, the area is continuously monitored by networks of geophysical instruments managed by Istituto Nazionale di Geofisica e Vulcanologia (INGV) - Osservatorio Vesuviano. The tiltmeter array deployed at Campi Flegrei is part of the wider network designed to monitor the ground tilt of Neapolitan District volcanoes (Vesuvius, Campi Flegrei and Ischia). The network was originally made up of horizontal optical pendulums that recorded analog signals but later on these instruments were replaced by electronic sensors (bubble-tiltmeters). A qualitative leap took place when wells of $20-25 \mathrm{~m}$ of depth were drilled to anchor the cased digital sensors at their bottom. The current tiltmeter network at Campi Flegrei (Fig. 1) consists of ten instruments of three different types: four surface short baselength platform AGI 702 tiltmeters, three AGI 722 tiltmeters installed in shallow wells, and three borehole digital Lily tiltmeters. Signals acquired by surface tilt sensors can be significantly conditioned by temperature range; in particular, bubble-tiltmeters are very sensitive to daily thermal variations whose source is the solar radiation heating the ground not uniformly. In order to reduce the thermal excursions and the background noise on tilt signals, the three digital borehole instruments (CMP, ECO and HDM) were installed at average depths of $25 \mathrm{~m}$, where the temperature fluctuations are on the order of $0.01 \div 0.02^{\circ} \mathrm{C}$ (Aquino et al., 2016; Ricco et al., 2018). These tiltmeter stations consist of digital sensors model "Lily Self-Leveling Borehole Tiltmeter" (Jewell Instruments ex AGI) equipped with a self-leveling bubble electrolyte on a range of $\pm 10^{\circ}$, with a dynamic range of $\pm 330 \mu$ radians and a resolution less than 5 nradians. The tiltmeter package includes a magnetic compass and temperature sensor (Jewell Instruments, 2013). Ground tilt variations are measured along two orthogonal directions NS and EW; they are recorded with a sampling rate of 1 sample per minute.

In the present work, we use tilt data recorded by the three borehole instruments since their installation in 2015 .

\section{Data analysis}

The past analyses of the ground tilt at Campi Flegei have evidenced that medium/long period tidal constituents induce 


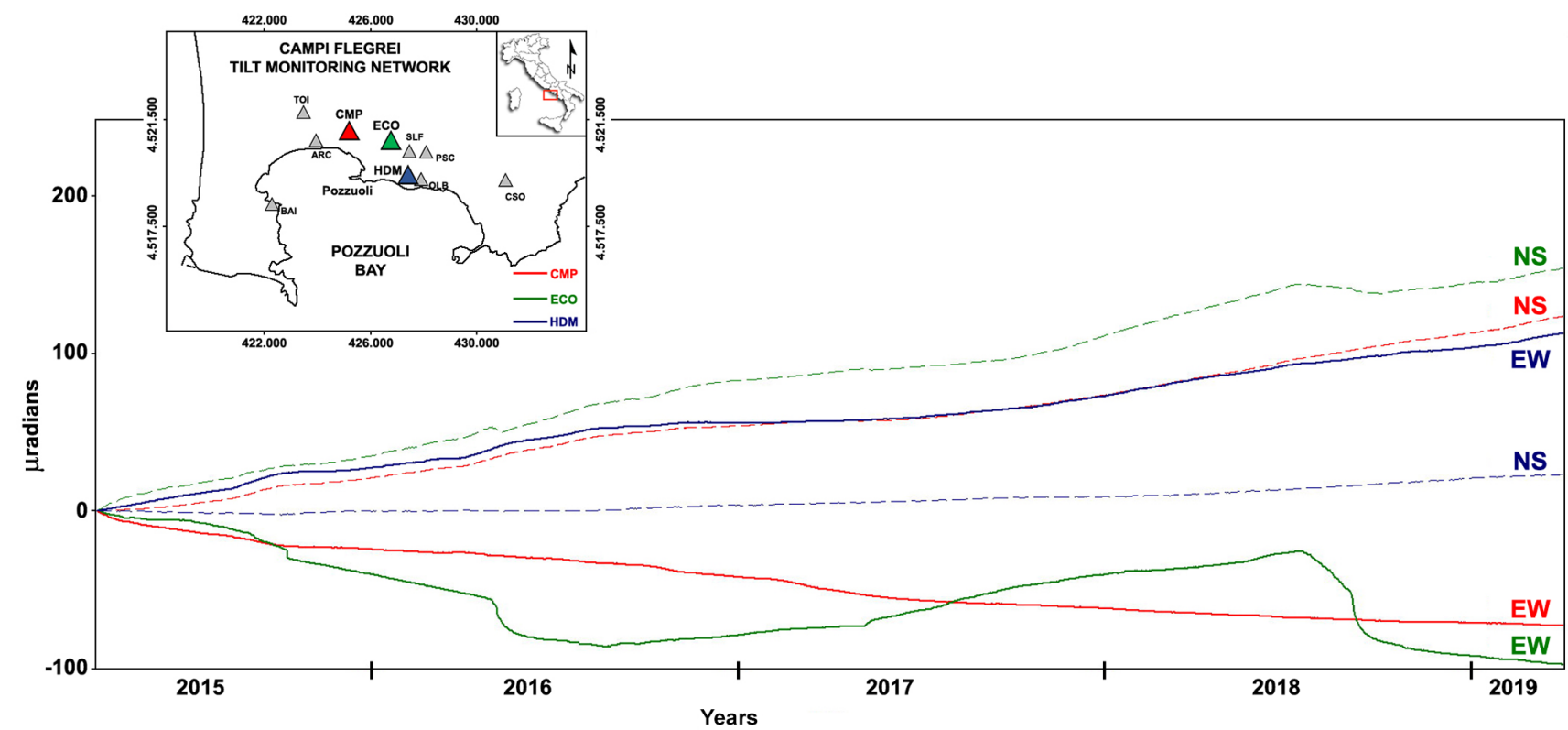

Figure 1. Map of the tiltmeter network at Campi Flegrei and ground tilt (raw signal) recorded in four years at CMP, ECO and HDM instruments. The colours identify the different sensors, dashed and continuous lines indicate the NS, EW components, respectively.

ground oscillations which are superimposed to the normal deformation trend of the area (De Lauro et al.; 2018; Ricco et al., 2019). In particular, the monthly Mm, fortnightly Mf and solar diurnal S1 constituents were recognized in tilt data by using the Independent Component Analysis (Bottiglieri et al., 2007, 2010, De Lauro et al., 2018). Some seasonal variations in the tiltmeter pattern were recognised over 21 months of data (29 March 2015-31 December 2016) by De Lauro et al. (2018); at the present, due to the availability of four years of data, we can go further and investigate the temporal pattern of ground deformation and its eventual seasonal variations more deeply. Thus, the tiltmeter time series recorded from 1 April 2015 to 31 March 2019 were filtered by using a bandpass acausal (zero phase) Butterworth filter of the third order. The filtering bands were centered on the periods of Mm $(T=27.55 \mathrm{~d})$, Mf $(T=13.66 \mathrm{~d})$ and S1 $(T=24 \mathrm{~h})$, with bandwidth equal to [23-31 d], [13-14 d] and [23.7-24.2 h], respectively. Given the filtered time series, the azimuth of the tilt vector was estimated over sliding time windows with length equal to the period of each constituent.

Looking at the filtered time series, we find clear evidence of the amplitude seasonal modulation for the diurnal S1 (Fig. 2). Interestingly, during the spring-summer time, the amplitude increase is particularly evident for the EW component of CMP and, to a lesser extent, for both the components of ECO. On the contrary, no evident variation occurs at HDM site. Thus, the results confirm that the occurrence of thermoelastic effects depends on the site, as hypothesized by De Lauro et al. (2018). These authors recognized that the local geology plays an important role in modulating the amplitude of the diurnal S1 constituent detected in the tilt time series. In fact, the diurnal S1 is strictly related to the insolation and induces a thermoelastic strain, whose propagation at depth is controlled by the rheology. The composite model proposed by Ben-Zion and Leary (1986) predicts that this strain is greater for unconsolidated heterogeneous materials. The seasonal behavior of the ground deformation at CMP, ECO and HDM sites is also reflected in the temporal pattern of the tilt azimuths which are more scattered during the fall-winter months compared with spring-summer time, when they attain stable values. However, despite these fluctuations, the average values of the azimuths retrieved in both fall-winter and spring-summer are comparable (see next analysis).

In order to determine the orientation of the main tilting planes and their possible seasonal variability, we investigated the statistical distribution of the azimuth values for the entire dataset 2015-2019, as well as for the sub-intervals that correspond to the wet and dry seasons of the hydrological year. Indeed, as already pointed out by Petrosino et al. (2018), at Campi Flegrei the hydrological year can be divided into two time intervals on the basis of the average intra-annual distribution of the rainfall: the dry season goes from April to August and the wet season from September to March. Therefore, our dataset encompasses exactly four dry (hereinafter, P1, P3, P5 and P7) and wet seasons (P2, P4, P6 and P8), according to Table 1.

Besides these intervals, we also considered the cumulative distribution of the azimuths over the four dry $\quad\left(\mathrm{P} \_\mathrm{DRY}=\mathrm{P} 1+\mathrm{P} 3+\mathrm{P} 5+\mathrm{P} 7\right)$ and wet 

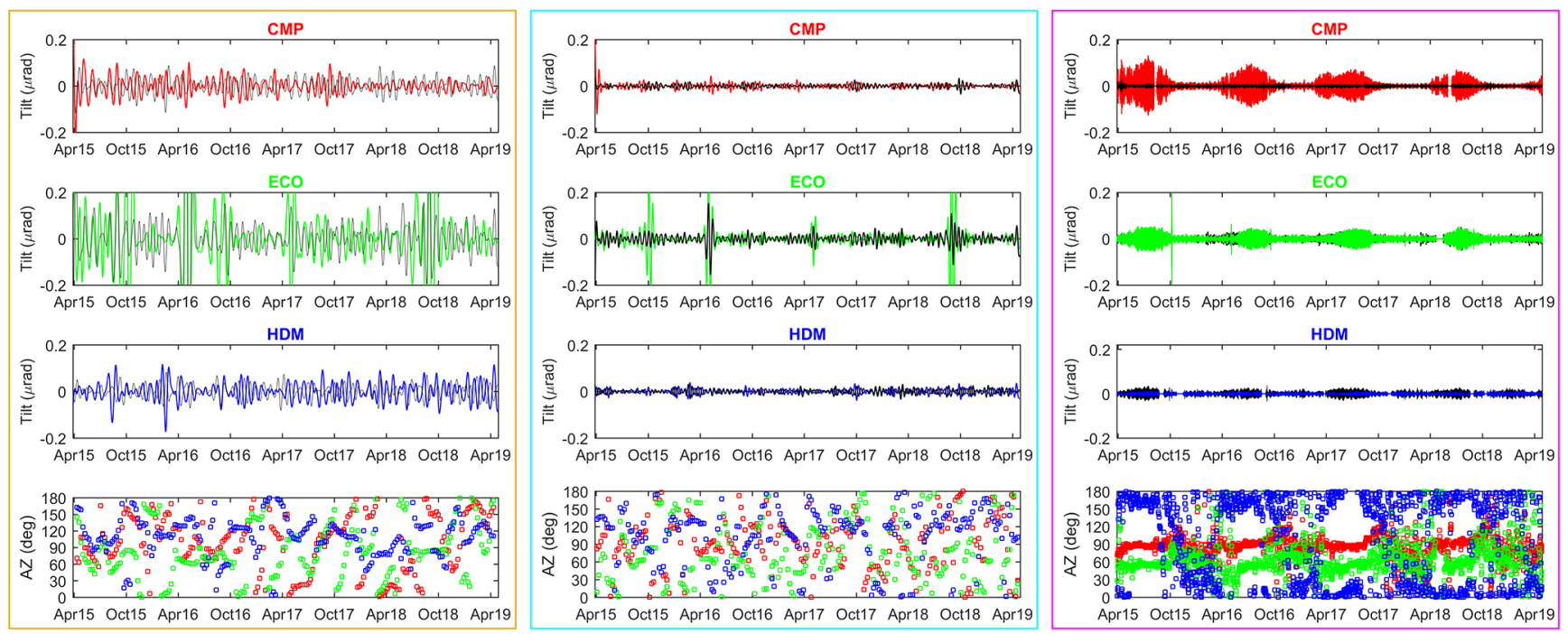

Figure 2. Time series of the two tiltmeter components (EW in red, green and blue for CMP, ECO and HDM, respectively; NS always in black for the three instruments) and temporal pattern of the azimuth. From left to right, the three boxes in ocra, cyan and magenta are relative to the results obtained in the period band corresponding to the Mm, Mf and S1 tidal constituents, respectively.

Table 1. Start and end times of the temporal intervals used for the partition of the dataset.

\begin{tabular}{lll}
\hline Time Interval & Start & End \\
\hline P1 - dry & 1 April 2015 & 31 August 2015 \\
P2 - wet & 1 September 2015 & 31 March 2016 \\
P3 - dry & 1 April 2016 & 31 August 2016 \\
P4 - wet & 1 September 2016 & 31 March 2017 \\
P5 - dry & 1 April 2017 & 31 August 2017 \\
P6 - wet & 1 September 2017 & 31 March 2018 \\
P7 - dry & 1 April 2018 & 31 August 2018 \\
P8 - wet & 1 September 2018 & 31 March 2019 \\
\hline
\end{tabular}

$\left(\mathrm{P} \_\mathrm{WET}=\mathrm{P} 2+\mathrm{P} 4+\mathrm{P} 6+\mathrm{P} 8\right) \quad$ seasons. The resulting rose plots are shown in Fig. 3.

Overall, the rose plots show that the resulting azimuth values range in a narrow interval, confirming as observed in De Lauro et al. (2018) and Ricco et al. (2019). Nevertheless, this analysis applied to 4 year-long dataset which allows finer investigation over smaller sub-intervals, brings out some additional outcomes, evidencing that in some cases a broader distribution is obtained.

Regarding the Mm constituent, we observe that at CMP site, the azimuths of the tilting plane over the whole investigated time range are quite disperse, with an average orientation roughly in the ESE-WNW direction. Indeed, this spread is mainly due to the difference between dry and wet seasons: in P_DRY the azimuth are oriented ENE-WSW, while in P_WET they are along SE-NW. For the Mf constituent, the average direction is nearly ESE-WNW, with dominant E-W orientation in P_DRY, and ESE-WNW in P_WET. The par- tition of the dataset into dry and wet seasons thus reveals that a rotation of the main tilt direction for both the $\mathrm{Mm}$ and $\mathrm{Mf}$ constituents occurs between the two hydrological seasons.

At ECO site, the tilting planes of the Mm and Mf tidal constituents are on the average oriented along the ENE-WSW direction. In P_DRY a fairly good collimation persists in the ENE-WSW; on the contrary, the cumulative distribution in $\mathrm{P}_{-}$WET is characterized by a wide dispersion of the azimuths.

At HDM site, the Mm and Mf azimuths exhibit the lowest degree of variability compared to the previous sites. Indeed, they are always oriented nearly ESE-WNW throughout the whole 4 year-long observational interval, with no difference between wet and dry seasons.

Regarding the diurnal S1 constituent, at CMP and ECO sites the azimuths are almost constant (nearly E-W and ENE-WSW, respectively), with no evident seasonal variations. At HDM the average direction is nearly $\mathrm{N}-\mathrm{S}$, with no appreciable differences between wet and dry seasons, except for a more dispersive pattern during the wet months.

In order to check whether the orientations of the tilt vectors are non-uniformly distributed, we adopted a statistical approach. In particular, we applied the Rayleigh test (Berens, 2009) that assumes as null hypothesis a uniform distribution of the sample populations around the circle. A significance level of 0.05 is usually considered: probability $(p)$ values lower than $5 \%(p<0.05)$ lead to reject the null hypothesis, meaning a significant departure from uniformity and thus, the existence of preferred orientation of the tilt azimuth (the ground tilting occurs on a preferential plane). On the contrary, $p$-values greater than $5 \%$ correspond to azimuthal uniform distribution (no preferential plane of ground tilting). 


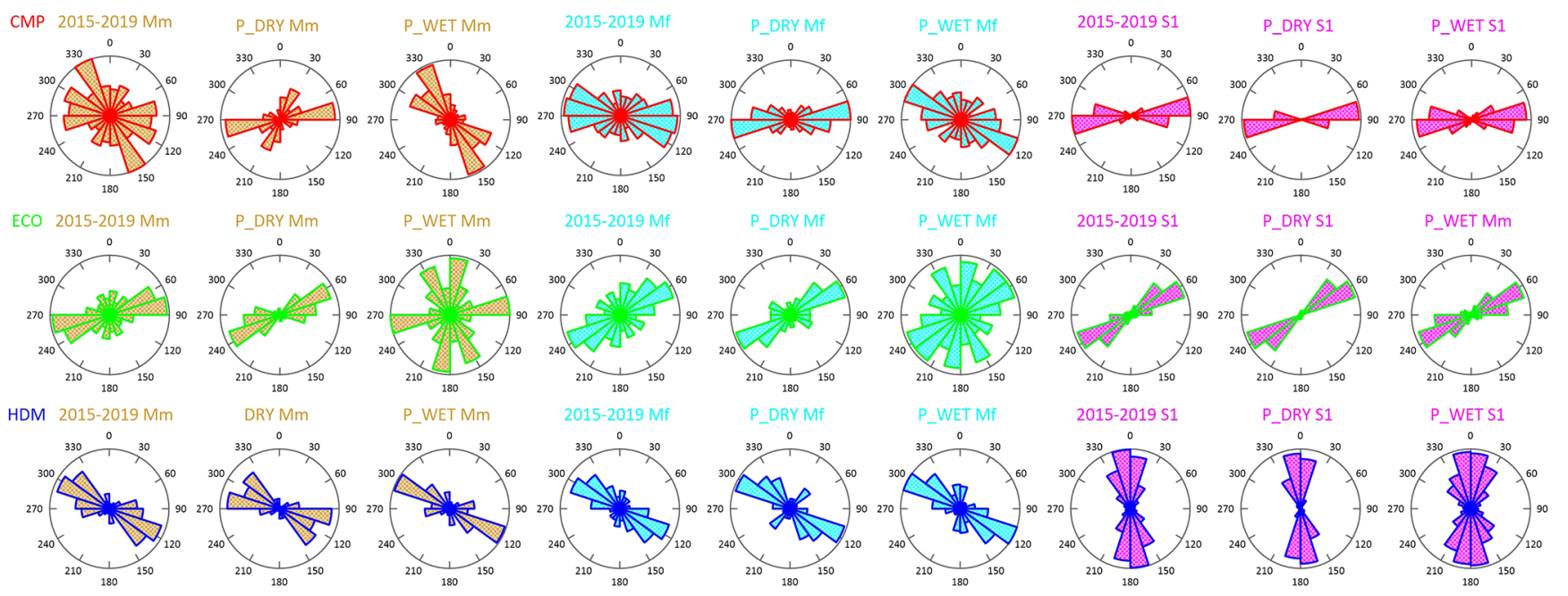

Figure 3. Rose diagrams of the azimuths of the tilt signals. Each row is relative to a tiltmeter (red $=\mathrm{CMP}$, green $=\mathrm{ECO}, \mathrm{blue}=\mathrm{HDM}$ ) and shows the azimuths retrieved in 2015-2019, P_DRY and P_WET time ranges for the Mm (ocre), Mf (cyan) and S1 (magenta) tidal constituents.

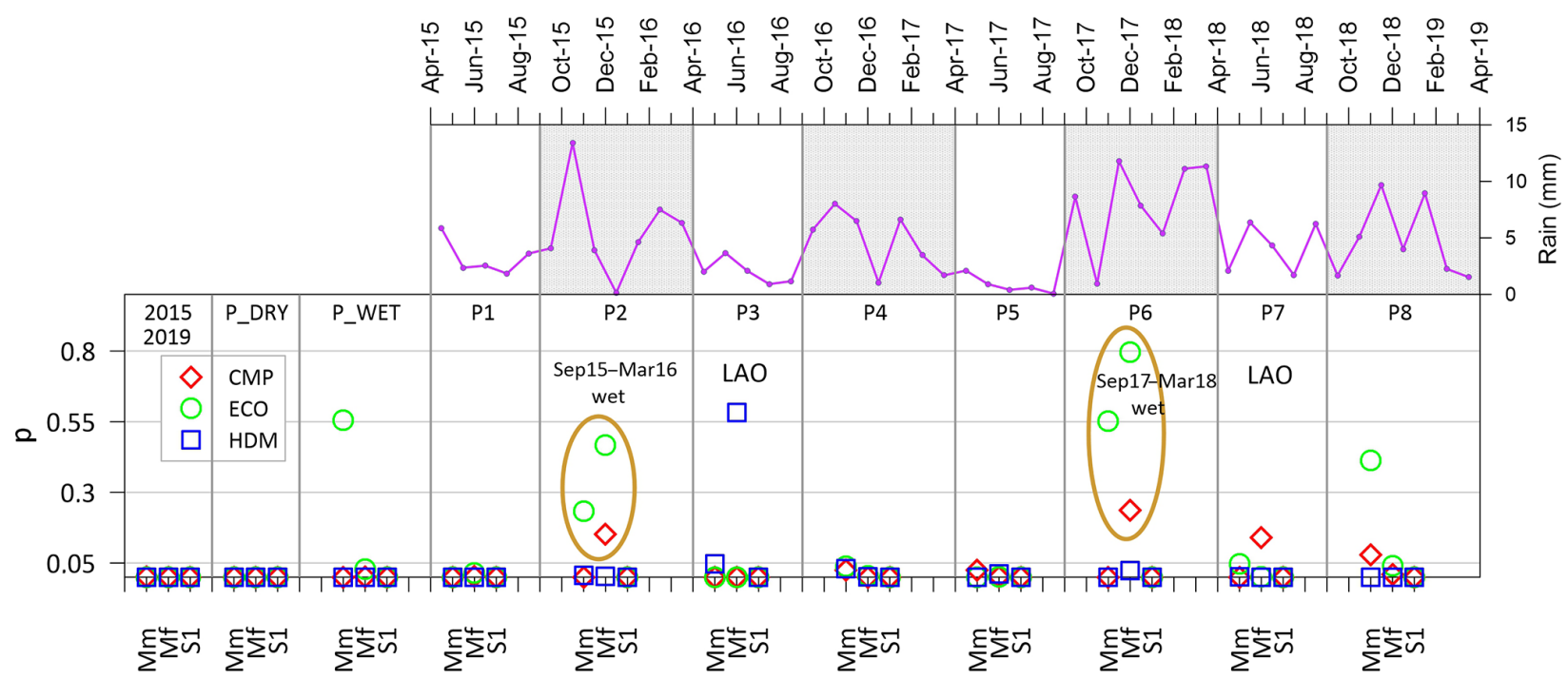

Figure 4. Probability ( $p$ ) values obtained from the Rayleigh test performed on the azimuthal distributions for the three tiltmeters. $p$-values lower than 0.05 corresponds to non-uniform distribution, while $p$-values greater than 0.05 support the hypothesis of uniformity. The different time intervals and tidal constituents are indicated on the top and bottom $x$ axes, respectively. The LAO occurrence is also annotated. In the upper panel, the monthly rainfall amount (purple line) is reported (data for Pozzuoli town, available at the URL https://www.3bmeteo.com/ meteo/pozzuoli/storico); in this plot, the light-grey boxes in the background mark the wet season of the hydrological year.

We tested the azimuth distribution considering: (a) the entire dataset, (b) each sub-interval related to dry and wet seasons (P1-P8), (c) the cumulative dry and wet seasons (P_DRY, P_WET). The results of the tests are summarized in Fig 4.

In general the azimuthal distributions for the different tiltmeters, constituents and time ranges are significantly nonuniform. However, in some cases (see e.g. the distribution in P_WET for the Mm at ECO) the null hypothesis of uniformity cannot be rejected. Going into details, from the rose plots (Fig. 3) relative to the entire dataset 2015-2019 and to the cumulative dry and wet seasons (P_DRY and P_WET), it emerges that at ECO site a common average azimuthal tilt direction along the ENE-WSW exists in all the three intervals for both $\mathrm{Mm}$ and Mf. However, in P_WET additional ground tilting along a nearly $\mathrm{N}-\mathrm{S}$ plane overlaps to the basic ENE-WSW tilt pattern, thus causing a departure from nonuniformity of the azimuth distributions.

Another feature attracts attention in Fig. 4: during P2, the Rayleigh test detects (with particularly high $p$-values) three contemporary relevant departures from non-uniformity for 
$\mathrm{Mm}$ and Mf, at ECO and CMP tiltmeters. This pattern recurs in a similar fashion in P6. It is noteworthy that both P2 and P6 wet seasons (September 2015-March 2016 and September 2017-March 2018, respectively) were characterized by a high rate of rainfall (Fig. 4; see also Fig. 8 in Ricco et al., 2019). This observation suggests that the connection between meteoric water amount and non-uniform azimuthal distributions should be deeply examined. Moreover, the dry seasons $\mathrm{P} 3$ and $\mathrm{P} 7$ which followed P2 and P6 were marked by the occurrence of two aseismic tilt reversal episodes (April 2016 and August 2018, respectively) associated with Large Amplitude Oscillations (LAO) of the Mf constituent, as recognized by Ricco et al. (2019). Thus, the departure from a non-uniform distribution of the azimuth (and the consequent trend to uniformity) seems to be a proxy of aseismic tilt reversal phenomena. Of course, this possible relationship deserves particular attention and further investigations on a longer time record.

\section{Discussion and conclusions}

Recent studies of the ground tilt at Campi Flegrei highlighted that the medium responds to a complex mixing of endogenous (e.g. hydrothermal, volcanic) and exogenous (e.g. tidal, meteoric) sources (Petrosino et al. 2018; De Lauro et al., 2018; Ricco et al., 2019). In particular, De Lauro et al. (2018) evidenced how earth tides induce ground oscillations along well defined planes whose orientations are constrained by the local stress field and by the distribution of faults and fractures. With the present research we go further, evidencing seasonal variations of the tidal tilting, which are ascribable to the rainfall. Indeed, water infiltration in the ground, according to the theory of poroelasticity (Wang, 2000; Wang and Kumpel, 2003), can contribute to modify the local stress field thus changing the observed deformation pattern (Dal Moro and Zadro, 1998; Weise et al., 1998; Dong et al., 2002; Uhlemann et al., 2016; Lesparre et al., 2017). In line with these studies, we interpret the observed variations on tidal scales in the tilt directions as an effect of the local geology, modulated by the rainfall seasonality. During the dry seasons, at CMP, medium/long period tidal stresses induce oscillations along well defined planes whose orientations (ENE-WSW for Mm and $\mathrm{E}-\mathrm{W}$ for Mf) are constrained by the rheology and local structure (De Lauro et al., 2018). In the wet seasons, the infiltration of meteoric water in the ground causes a variation of the pore pressure with a consequent modification of the stress field, in turn generating a rotation of the tilting planes. In addition, the presence of fractures loaded by infiltrated water could not be neglected in this "tilt switching" mechanism. In fact, as demonstrated by Lesparre et al. (2017), the activation of fluid-filled structures, which become the main source of deformation, can induce variations of the orientations of the tilting planes. In other words, it is like a new deformation source (on medium/long period tidal scales) "turns on" in the wet period and completely dominates over the source active in the dry season. A support for this hypothesis comes from the analysis of the vectorial plots (hodograph) of the unfiltered tilt data which represent the resultant kinematics due to the sum of internal (volcanic uplift) and external (tidal, meteoric) sources of deformation over different time scales. As example for the time intervals P5-P6 (Fig. 5), we observe at CMP a change in kinematics because the average preferential direction of the tilt trend shows a rotation moving from dry to wet season. This is a clear indication that the process that generates the switching of the azimuth of the tilt planes on the Mm and Mf tidal scales is so powerful to induce a sensible variation of the resultant kinematic pattern.

Regarding the time evolution of the tidal tilt induced by the $\mathrm{S} 1$ constituent, the investigation over the 4 year-long time span at CMP does not reveal any rotation of the azimuth between dry and wet seasons, but only an evident amplitude variation likely related to the local rheology consisting in layers of sands alternated to ashes and pyroclastics (Aquino et al., 2016). In the framework of the thermoelastic model (Ben-Zion and Leary, 1986), the loose and unconsolidated soils would thus cause the observed amplitude amplification and thermoelastic site effects (De Lauro et al., 2018).

On medium/long period tidal scales, ECO site shows an even more complex behaviour compared with CMP: in the dry seasons a preferred orientation (ENE-WSW) of the tilting planes exists, compatible with the existence of a main source of deformation. In the wet seasons, this pattern is apparently lost because we observe a uniform distribution of the azimuths around the circle. We can hypothesize that this distribution is due to the superposition of at least two (or even more) tilting planes. This would mean, for example, that the rainfall infiltration activates, by a mechanism of water loading, fractures which act as additional sources of deformation. Thus, during the wet seasons, coexisting multiple sources likely leads to the breaking of the non-uniformity of the distribution of the tilting azimuths usually observed in the dry seasons. Also in the case of ECO, the effects of this phenomenon can be noticed in the resultant kinematics of the tilt vector. In fact, the average tilt direction of the unfiltered data shows a rotation moving from dry to wet seasons, as shown in Fig. 5 for the P5-P6 time interval.

As for CMP site, also at ECO the tilting azimuth of the S1 tidal constituent is stable over dry and wet seasons, with a moderate amplitude variation. The observed thermoelastic site effects (De Lauro et al., 2018) are related to the presence of a sequence of pyroclastic deposits with various granulometry (Aquino et al., 2016).

Finally, HDM shows a completely different behavior compared with CMP and ECO sites. In fact both the directions of the tilting planes and the amplitudes of the ground deformation in all the three analyzed tidal band Mm, Mf and S1, are nearly constant throughout the investigated 4 year-long time interval, without any substantial change between dry and wet seasons. The absence of both azimuthal and amplitude vari- 


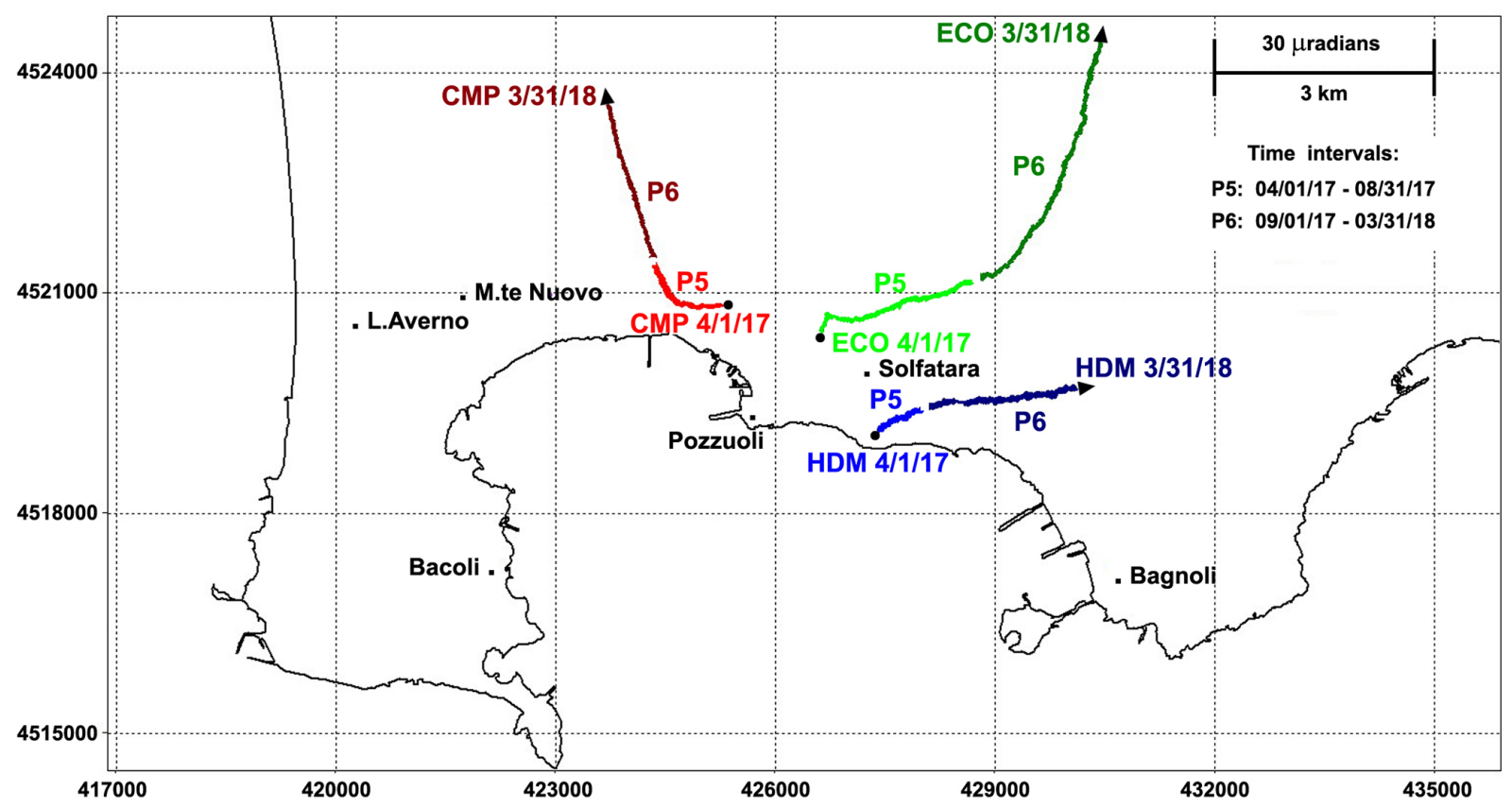

Figure 5. Plots of the tilt vectors at the three sites CMP, ECO and HDM. The curves that originate from each station indicates the cumulative tilt variation (hodograph) recorded in P5 = 1 April 2017-31 August 2017 (light color) and P6 = 1 September 2017-31 March 2018 (dark color). Each mesh is equivalent to a tilt variation of $30 \mu$ radians. The boundary between the two intervals P5 and P6 is marked with a white square.

ations is likely due to the specific nature of the trachytic lava dome of Mt. Olibano, where the borehole tiltmeter HDM is installed (Aquino et al., 2016). In fact, the site is characterized by lava layers with thicknesses of about $4-5 \mathrm{~m}$, intercalated by pyroclastics and sands. The porosity of this igneous rock is certainly lower than that of the pyroclastic deposits at ECO and CMP sites. HDM site therefore seems "insensitive" to the rain because there are no relevant poroelastic effects and/or fracture filling phenomena that would in turn generate additional seasonal sources of deformation. Moreover, while the unconsolidated soils of CMP and ECO sites tend to amplify the ground tilt related to the diurnal S1 constituent, the harder lava rocks at HDM act as a damper, thus minimizing thermoelastic site effects (De Lauro et al., 2018).

In conclusion, we can hypothesize that the deformation detected at Campi Flegrei by the tiltmeters is due to the effects of different processes. The main process concerns the renewed uplift which since 2004 has been involving this area: the observed tilting direction is coherent with the ground deformation pattern generated by a centroid (center of pressure) located at Pozzuoli. This first-order effect is accompanied, more or less intensely, by the medium response to the tidal stresses. The tidal tilting is essentially controlled by rheology, fracture distribution and local stress field. The latter is strongly dependent on the underground water whose mobility (which in turn depends on pressure variations and rainfall amount) triggers, with seasonal periodicity, secondorder local effects consisting of small ground deformation.
These secondary sources can induce the observed variations of the tilting directions at level of the medium/long period tidal components ( $\mathrm{Mm}$ and $\mathrm{Mf}$ ) and contribute to modify the local tilt kinematics. Structural features also affect the amplitude of tilt oscillations related to the diurnal S1 constituent, leading to thermoelastic site effects.

The present study provides a key for better understanding the complex mixing of sources of deformation which act at Campi Flegrei caldera. The identification of main and secondary ground deformation processes through the analysis of their effects on the tiltmeter signals surely represents the first step for the definition of the background state. In fact, the recognition in the tilt pattern of seasonal tidal effects, superimposed to the normal trend related to endogenous phenomena, is crucial: focusing on the internal sources after having removed external factors allows a better comprehension of the volcanic dynamics of the caldera. On the other hand, the study of these secondary effects due to exogenous sources like tides and rainfall is useful to identify and locate structural features (faults and fractures) and to provide constraints on both their geometry and rock properties of the surrounding medium, thus improving the knowledge of the local geology. In this perspective, future efforts should be done towards the development of a quantitative model to study fluidcoupled processes modulated by the external factors. Assessing how this interaction modifies the local stress field would shed more light on the synchronization mechanisms between ground deformation and earth tides. 
Data availability. Data are available on request from the authors.

Author contributions. SP, CR, EDL, MF conceived the original idea of the present research and developed new methodological approaches for tiltmeter data analysis. SP, EDL, MF, CR, IA elaborated and validated the data. All the authors contributed in writing and reviewing the manuscript.

Competing interests. The authors declare that they have no conflict of interest.

Special issue statement. This article is part of the special issue "Understanding volcanic processes through geophysical and volcanological data investigations: some case studies from Italian sites (EGU2019 GMPV5.11 session, COV10 S01.11session)". It is not associated with a conference.

Acknowledgements. The authors wish to thank Vincenzo Augusti for providing regular maintenance of the tiltmeter network, and two anonymous referees for their useful comments that contributed to improve this manuscript.

Review statement. This paper was edited by Danilo Galluzzo and reviewed by two anonymous referees.

\section{References}

Aquino, I., Ricco, C., Del Gaudio, C., Augusti, V., and Scarpato, G.: Potenziamento delle Reti Tiltmetriche nell'area vulcanica campana: rapporto sull'attività svolta nell'ambito del progetto vulcamed, Rapporti Tecnici INGV, 348, ISSN2039-6651, INGV, Rome, Italy, 2016.

Ben-Zion, Y. and Allam, A. A.: Seasonal thermoelastic strain and postseismic effects in Parkfield borehole dilatometers, Earth Planet. Sc. Lett., 379, 120-126, https://doi.org/10.1016/j.epsl.2013.08.024, 2013.

Ben-Zion, Y. and Leary, P.: Thermoelastic strain in a half-space covered by unconsolidated material, B. Seismol. Soc. Am., 76, 1447-1460, 1986.

Berens, P.: CircStat: a Matlab toolbox for circular statistics, J. Stat. Softw., 31, 1-21, 2009.

Bottiglieri, M., Falanga, M., Tammaro, U., Obrizzo, F., De Martino, P., Godano, C., and Pingue, F.: Independent component analysis as a tool for ground deformation analysis, Geophys. J. Int., 168, 1305-1310, https://doi.org/10.1111/j.1365-246X.2006.03264.x, 2007.

Bottiglieri, M., Falanga, M., Tammaro, U., De Martino, P., Obrizzo, F., Godano, C., and Pingue, F.: Characterization of GPS time series at the Neapolitan volcanic area by statistical analysis, J. Geophys. Res., 115, B10416, https://doi.org/10.1029/2009JB006594, 2010.
Capuano, P., De Lauro, E., De Martino, S., Falanga, M., and Petrosino, S.: Convolutive independent component analysis for processing massive datasets: a case study at Campi Flegrei (Italy), Nat. Hazards, 86, 417-429, https://doi.org/10.1007/s11069-0162545-0, 2017.

Cusano, P., Petrosino, S., De Lauro, E., and Falanga, M. The whisper of the hydrothermal seismic noise at Ischia Island, J. Volcanol. Geoth. Res., 389, 106693, https://doi.org/10.1016/j.jvolgeores.2019.106693, 2020.

Dal Moro, G. and Zadro, M.: Subsurface deformations induced by rainfall and atmospheric pressure: tilt/strain measurements in the NEItaly seismic area, Earth Planet. Sc. Lett., 164, 193-203, https://doi.org/10.1016/S0012-821X(98)00203-9, 1998.

De Lauro, E., Falanga, M., and Petrosino, S.: Study on the longperiod source mechanism at Campi Flegrei (Italy) by a multiparametric analysis, Phys. Earth Planet. In., 206-207, 16-30, https://doi.org/10.1016/j.pepi.2012.06.006, 2012.

De Lauro, E., De Martino, S., Falanga, M., and Petrosino, S.: Synchronization between tides and sustained oscillations of the hydrothermal system of Campi Flegrei (Italy), Geochem. Geophy. Geosy., 14, 2628-2637, https://doi.org/10.1002/ggge.20149, 2013.

De Lauro, E., De Martino, S., Falanga, M., and Petrosino, S.: Fast wavefield decomposition of volcano-tectonic earthquakes into polarized $\mathrm{P}$ and $\mathrm{S}$ waves by independent component analysis, Tectonophysics, 690, 355-361, https://doi.org/10.1016/j.tecto.2016.10.005, 2016.

De Lauro, E., Petrosino, S., Ricco, C., Aquino, I., and Falanga, M.: Medium and long period ground oscillatory pattern inferred by borehole tiltmetric data: New perspectives for the Campi Flegrei caldera crustal dynamics, Earth Planet. Sc. Lett., 504, 21-29, https://doi.org/10.1016/j.epsl.2018.09.039, 2018.

Del Gaudio, C., Aquino, I., Ricciardi, G.P., Ricco, C., and Scandone, R.: Unrest episodes at Campi Flegrei: a reconstruction of vertical ground movements during 1905-2009, J. Volcanol. Geoth. Res., 195, 48-56, https://doi.org/10.1016/j.jvolgeores.2010.05.014, 2010.

De Martino, P., Tammaro, U., and Obrizzo, F.: GPS time series at Campi Flegrei caldera (2000-2013), Ann. Geophys.-Italy, 57, 0213, https://doi.org/10.4401/ag-6431, 2014.

De Martino, S., Palo, M., and Cimini, G. B.: A statistical study of the Stromboli volcano explosion quakes before and during 2002-2003 eruptive crisis, J. Geophys. Res., 116, B04303, https://doi.org/10.1029/2010JB008047, 2011a.

De Martino, S., Falanga, M., Palo, M., Montalto, P., and Patané, D.: Statistical analysis of the volcano seismicity during the 2007 crisis of Stromboli, Italy, J. Geophys. Res., 116, B09312, https://doi.org/10.1029/2010JB007503, 2011 b.

Dong, D., Fang, P., Bock, Y., Cheng, M. K., and Miyazaki, S.: Anatomy of apparent seasonal variations from GPSderived site position time series, J. Geophys. Res., 107, 2075, https://doi.org/10.1029/2001JB000573, 2002.

Fabian, M. and Kümpel, H. J.: Poroelasticity: observations of anomalous near surface tilt induced by ground water pumping, J. Hydrol., 281, 187-205, https://doi.org/10.1016/S00221694(03)00234-8, 2003.

Falanga, M. and Petrosino, S.: Inferences on the source of longperiod seismicity at Campi Flegrei from polarization analysis 
and reconstruction of the asymptotic dynamics, B. Volcanol., 74, 1537-1551, https://doi.org/10.1007/s00445-012-0612-2, 2012.

Jewell Instruments: LILY Self-Leveling Borehole Tiltmeter, User's Manual No. B-05-1003, Rev. G., San Francisco, USA, 2013.

Kümpel, H. J., Lehmann, K., Fabian, M., and Mentes, G.: Point stability at shallow depths: experience from tilt measurements in the Lower Rhine Embayment, Germany, and implications for high-resolution GPS and gravity recordings, Geophys. J. Int., 146, 699-713, https://doi.org/10.1046/j.1365246X.2001.00494.x, 2001.

Lesparre, N., Boudin, F., Champollion, C., Chery, J., Danquigny, C., Seat, H. C., Cattoen, M., Lizion, F., and Longuevergne, L.: New insights on fractures deformation from tiltmeter data measured inside the Fontaine de Vaucluse karst system, Geophys. J. Int., 208, 1389-1402, https://doi.org/10.1093/gji/ggw446, 2017.

McNutt, S. R. and Beavan, R. J.: Volcanic earthquakes at Pavlof volcano correlated with the solid earth tide, Nature, 294, 615618,1981

Petrosino, S., Cusano, P., and Madonia, P.: Tidal and hydrological periodicities of seismicity reveal new risk scenarios at Campi Flegrei caldera, Sci. Rep.-UK, 8, 13808, https://doi.org/10.1038/s41598-018-31760-4, 2018.

Queitsch, M., Jentzsch, G., Weise, A., Ishii, H., and Asai, Y.: Pumping induced pore pressure changes in tilt measurements near a fault zone in Mizunami, Japan, in: Proceedings of the International Association of Geodesy Symposia, 139, 113-118, Springer, Berlin, Germany, 2014.

Ricco, C., Aquino, I., Augusti, V., D’Auria, L., Del Gaudio, C., and Scarpato, G.: Improvement and development of the tiltmetric monitoring networks of Neapolitan volcanoes, Ann. Geophys.Italy, 61, SE114, https://doi.org/10.4401/ag-7496, 2018.

Ricco, C., Petrosino, S., Aquino, I., Del Gaudio, C., and Falanga, M.: Some Investigations on a Possible Relationship between Ground Deformation and Seismic Activity at Campi Flegrei and Ischia Volcanic Areas (Southern Italy), Geosciences, 9, 222, https://doi.org/10.3390/geosciences9050222, 2019.
Saccorotti, G., Petrosino, S., Bianco, F., Castellano, M., Galluzzo, D., La Rocca, M., Del Pezzo, E., Zaccarelli, L., and Cusano, P.: Seismicity associated with the 2004-2006 renewed ground uplift at Campi Flegrei Caldera, Italy, Phys. Earth Planet. In., 165, 14 24, https://doi.org/10.1016/j.pepi.2007.07.006, 2007.

Uhlemann, S., Smith, A., Chambers, J., Dixon, N., Dijkstra, T., Haslam, E., Meldruma, P., Merritt, A., Gunn, D., and Mackay, J.: Assessment of ground-based monitoring techniques applied to landslide investigations, Geomorphology, 253, 438-451, https://doi.org/10.1016/j.geomorph.2015.10.027, 2016.

Wang, H. F.: Theory of Linear Poroelasticity with Applications to Geomechanics and Hydrogeology, Princeton University Press, Princeton, USA, p. 287, 2000.

Wang, R. and Kümpel, H. J.: Poroelasticity: efficient modeling of strongly coupled slow deformation processes in multi-layered half-space, Geophysics, 68, 705-717, https://doi.org/10.1190/1.1567241, 2003.

Weise, A., Jentzsch, G., Kiviniemi, A., and Kääriäinen, J.: Comparison of long-period tilt measurements: results from the two clinometric stations Metsähovi and Lohja, Finland, J. Geodyn., 27, 237-257, https://doi.org/10.1016/S0264-3707(97)00067-7, 1998.

Westerhaus, M. and Welle, W.: Environmental effects on tilt measurements at Merapi volcano, Bull. Inf. Marees Terr., 137, 10917-10926, 2002.

Westerhaus, M., Altmann, J., and Heidbach, O.: Using topographic signatures to classify internally and externally driven tilt anomalies at Merapi Volcano, Java, Indonesia, Geophys. Res. Lett., 35, L05310, https://doi.org/10.1029/2007GL032262, 2008.

Wyatt, F. and Berger, J.: Investigations of tilt measurements using shallow borehole tiltmeters, J. Geophys. Res.-Sol Ea., 85, 43514362, https://doi.org/10.1029/JB085iB08p04351, 1980. 